\title{
INSULIKE Tablets: A Phenotypic Nutrition for Natural Sugar Balance
}

Research Article

Govind Shukla, Monica Yadav, J.Sai Prasanna, C.J.Sampath Kumar

Lactonova Nutripharm (P) Ltd., Makers of INSULIKE Tablets, 81/3, IDA Mallapur, Hyderabad, Telangana, India-500 076.

Corresponding Author Author Email:

lactonovaresearch44@gmail.com

\section{INTRODUCTION}

Phenotypic Nutrition, A powerful strategy called phenotypic nutrition can help modulate the expression of your unique code, thus dramatically reducing your risk of developing disease. Phenotypic nutrition uses specific nutrients with the biochemical \& genetic effects to help protects the body from Diseases.

Diabetes is a multifactorial
Abstract: Diabetes is a multifactorial disease leading to several complications, and therefore demands a multiple therapeutic approach. Patients of diabetes either do not make enough insulin or their cells do not respond to insulin. In case of total lack of insulin, patients are given insulin injections. Whereas in case of those where cells do not respond to insulin many different drugs are developed taking into consideration possible disturbances in carbohydrate-metabolism. For example, to manage post-prandial hyper-glycaemia at digestive level, glucosidase inhibitors such as acarbose, miglitol and voglibose are used. These inhibit degradation of carbohydrates there by reducing the glucose absorption by the cells. To enhance glucose uptake by peripheral cells biguanide such as metformin is used. Sulphonylureas, like glibenclamide, is insulinotropic and work as secretogogue for pancreatic cells. Although several therapies are in use for treatment, there are certain limitations due to high cost and side effects such as development of hypoglycemia, weight gain, gastrointestinal disturbances, liver toxicity etc. Based on recent advances and involvement of oxidative stress in complicating diabetes mellitus, efforts are on to find suitable antidiabetic and antioxidant therapy. Medicinal plants are being looked upon once again for the treatment of diabetes. Many conventional drugs have been derived from prototypic molecules in medicinal plants. Metformin exemplifies an efficacious oral glucose-lowering agent. To date, over 400 traditional plant treatments for diabetes have been reported, although only a small number of these have received scientific and medical evaluation to assess their efficacy. The hypoglycemic effect of some herbal extracts has been confirmed in human and animal models of type 2 diabetes. The World Health Organization Expert Committee on diabetes has recommended that traditional medicinal herbs be further investigated. The present paper Reviews the Role of Insulike tablets developed by R\&D cell of Lactonova Nutripharm Pvt Ltd. Hyderabad in the role of herbal nutraceutical drug insulike, A nutriphenotypic approach for the treatment of Diabetes.

Keywords: Diabetes, Herbal nutraceuticals, Insulike tablet.

disease leading to several complications, and therefore demands a multiple therapeutic approach. Patients of diabetes either do not make enough insulin or their cells do not respond to insulin. In case of total lack of insulin, patients are given insulin injections. Whereas in case of those where cells do not respond to insulin many different drugs are developed taking into consideration possible disturbances in carbohydrate-metabolism. For example, to manage post-prandial hyper-glycaemia at digestive level, glucosidase inhibitors such as acarbose, miglitol and voglibose are used. These inhibit degradation of carbohydrates there by reducing the glucose absorption by the cells. To enhance glucose uptake by peripheral cells biguanide such as metformin is used. Sulphonylureas, like glibenclamide, is insulinotropic and work as secretogogue for pancreatic cells. Although several therapies are in use for treatment, there are certain limitations due to high cost and side effects such as development of hypoglycemia, weight gain, gastrointestinal disturbances, liver toxicity etc. Based on recent advances and involvement of oxidative stress in complicating diabetes mellitus, efforts are on to find suitable antidiabetic and antioxidant therapy. Medicinal plants are being looked upon once again for the treatment of diabetes. Many conventional drugs have been derived from prototypic molecules in medicinal plants. Metformin exemplifies an 
efficacious oral glucose-lowering agent. To date, over 400 traditional plant treatments for diabetes have been reported, although only a small number of these have received scientific and medical evaluation to assess their efficacy. The hypoglycemic effect of some herbal extracts has been confirmed in human and animal models of type 2 diabetes. The World Health Organization Expert Committee on diabetes has recommended that traditional medicinal herbs be further investigated.

\section{DIABETES MELLITUS - PATHOPHYSIOLOGY}

It is a disorder of carbohydrate metabolism wherein there is abnormal rise in blood glucose due to lack of insulin or lack of insulin resistance or a combination of both factors.

\section{Diabetes mellitus is of two types:}

Type-1: Insulin Dependant Diabetes Mellitus (IDDM)-In this condition, there is no production of insulin by the pancreas and the patient is totally dependant upon externally administered insulin. Type 1 diabetes is primarily due to autoimmune-mediated destruction of pancreatic $\beta$-cell islets, resulting in absolute insulin deficiency. People with type 1 diabetes must take exogenous insulin for the survival to prevent the development of ketoacidosis. Its frequency is low related to type 2 diabetes, which accounts for $90 \%$ of the case globally.

Type-2: Non-Insulin Dependant Diabetes Mellitus (NIDDM)-This condition is caused by the following factors:-

a) Insufficient production of insulin from pancreas.

b) Peripheral resistance by the cells to the action of available insulin.

c) Both a and b.

Type 2 diabetes is characterized by insulin resistance and/or abnormal insulin secretion, either of which may predominat. People of type 2 diabetes are not dependant on exogenous insulin, but may require it for controlled blood glucose levels if this is not achieved with diet alone or with oral hypoglycemic agents. The epidemic diabetes relates particularly to type 2 diabetes.

\section{EPIDEMIOLOGICAL FACTS}

Diabetes mellitus is caused by pronounced changes in human environment, and in human behavior and lifestyle, which have been a part and parcel of globalization, and these have resulted in escalating rates of both obesity and diabetes. Hence the recent adoption of the term "diadesity" was first suggested by Shafrir several decades ago.

Table: Number of people with diabetes (in millions) and the percentage increase over last 10 years.

\begin{tabular}{|l|c|c|c|}
\hline & Year-2000 & Year-2010 & \% Increase \\
\hline World & 15.1 & 22.1 & $46 \%$ \\
\hline North America & 14.2 & 17.5 & $23 \%$ \\
\hline South America & 15.6 & 22.5 & $44 \%$ \\
\hline Africa & 9.4 & 14.1 & $50 \%$ \\
\hline Europe & 26.5 & 32.9 & $24 \%$ \\
\hline Asia & 84.5 & 132.3 & $57 \%$ \\
\hline Australia & 1.0 & 1.3 & $33 \%$ \\
\hline
\end{tabular}


The global figure of people with diabetes is set to rise from current estimate of 150 millions to 220 millions in 2010, and 300 million in 2025. Most cases will be on type 2 diabetes, which is strongly assosiated with sedentary lifestyle and obesity.

\section{AETIOLOGICAL DETERMINANTS AND RISK FACTORS OF TYPE 2 DIABETES}

Genetic factors

$>$ Genetic markers, Family history, "Thrifty gene(s)".

Demographic characteristics

Sex, age, ethnicity.

Behavioral -lifestyle-related risk factors

$>$ Obesity (including distribution of obesity and duration).

$>$ Physical inactivity.

$>$ Diet.

$>$ Stress.

> "Westernization, urbanization, modernization".

Metabolic determinants and intermediate risk categories of type 2 diabetes

$>$ Impaired glucose tolerance.

$>$ Insulin resistance.

$>$ Pregnancy-related determinants (parity, gestational diabetes, diabetes in offspring of women with diabetes during pregnancy).

\section{INSULIKE Tablets: A Nutriphenotypic Approach for prevention \& Treatment of Diabetes}

A Nutriphenotyphic Approach. Insulike is an Ideal Regimen to fight against the single \& most important metabolic disease which can affect nearly every organ system in the body.

It has the power to protect from metabolic syndrome / type 2 diabetes mellitus. Few ingredients in insulike beneficially influence the expression of your genetic code $\&$ biochemical make up to decrease the disease risk. Insulike help to support the benefits of controlling Diabetes beyond glucose metabolism.,Inhibits glucose absorption,Stimulates insulin Secretion,Improves insulin binding,Improves capillary function,Prevents Pufa peroxidation \& also improves insulin sensitivity.

\section{Composition of insulike tablet}

\section{Each tablet contains:}

Eugenia Jambolana extract $-100 \mathrm{mg}$ 
Curcuminoids $-50 \mathrm{mg}$

Berberine Hydrochloride - 50mg

Mulberry leaf extract (4:1) - 33.5mg

Cynarins (from artichoke leaf extract) $-5 \mathrm{mg}$

Banaba leaf extract $-12 \mathrm{mg}$

L-Glutathione $-2.5 \mathrm{mg}$

Gymneic acids (from gymnema sylvestre extract) - 10mg

Charatin (from bitter milon extract) - 10mg

Methyl hydroxyl Chalcone polymers (from cinnamon extract) - 6.25mg

4-Hydroxy Isoleucine (from fenugreek) - 1.25mg

Anthocyanins (from bilberry fruit extract) $-3.10 \mathrm{mg}$

Epigallocatechin gallate (EGCG) from Green Tea Extract - 10mg

Alpha lipoic acid -100mg

Chromium (as chromium picolinate) - 40mcg

Selenium (as L-Selenomethionine) - 55mcg

Magnesium - 40mg

Vitamin C - 40mg

Vitamin D3 - 4000 IU

Methylcobalamin - 500mcg

Vitamin E - 12mg

Vitamin $\mathrm{K}-30 \mathrm{mcg}$

Vanadium - $1 \mathrm{mg}$

Biotin $-50 \mathrm{mcg}$

Benfotamine $-25 \mathrm{mg}$

Isoleucine $-25 \mathrm{mg}$

Glycine $-25 \mathrm{mg}$

$\mathrm{N}$-Acetyl cysteine $-25 \mathrm{mg}$

Other Ingredients: Eugenia Jambolana extract, Curcuminoids, Berberine Hydrochloride, Mulberry leaf extract, Artichoke leaf extract, Banaba leaf extract, L-Glutathione, Gymnema sylvestre extract, Bitter melon extract, 
Cinanmon extract, Fenugreek, Bilberry fruit extract, Green tea extract, Amino acids, Vitamins \&amp;amp; Minerals, Dibasic calcium phosphate [341(ii)], Microcrystalline cellulose [460(i)], Povidone [1201], Croscamellose Sodium [468], Magnesium Stearate [470(iii)], Silicon dioxide, Talc [553(iii)], Coating: Hydroxypropylmethylcellulose[464], Hydroxypropylcellulose[463], Titanium Dioxide (171), Iron Oxide Red [172 (ii)], Iron Oxide Yellow[172 (iii)], Polyethylene glycol [1521].

\section{ANTIDIABETIC ACTIVITY OF ACTIVE INGREDIENTS IN INSULIKE}

- MHCP (Methyl Hydroxy Chalcone Polymer): MHCP stimulates glucose uptake and glycogen synthesis to a similar level as insulin. In addition, MHCP treatment activated glycogen synthase and inhibited glycogen synthase kinase-3 $\beta$ activities, known effects of insulin treatment. Analysis of the insulin receptor demonstrated that the receptor was phosphorylated upon exposure to the MHCP. This supports that the insulin cascade was triggered by MHCP. Along with comparing MHCP to insulin, experiments were done with MHCP and insulin combined. The responses observed using the dual treatment were greater than additive, indicating synergism between the two compounds.these results demonstrate that the MHCP is an effective mimetic of insulin. MHCP may be useful in the treatment of insulin resistance and in the study of the pathways leading to glucose utilization in cells.

- Banaba leaf Extract (carosolic acid2\%): Banaba, also known as crepe myrtle, grows widely in tropical areas throughout India and Southeast Asia. For centuries, leaves have brewed as a tea for the treatment for diabetes. a from banaba, modern research has elucidated a probable mechanism of action. Stored within the cells of the body are specialized carrier proteins known as glucose transporter proteins, or GLUT. As the name suggests, glucose transporter proteins transport glucose from the blood into the cells. One type of GLUT transporter, known as GLUT4, is present in fat cells, skeletal muscle cells and cardio-muscle cells. GLUT4 migrates; it can either "hide" inside the cell, or it can be out in the open on the cell membrane. Exactly where GLUT4 happens to be determines how much glucose can enter the cell. If a lot of GLUT4 transporters are waiting at the cell membrane "doors," they can let glucose in. But if most of them are sequestered away in the inner part of the cell, glucose gets locked out. So what determines where GLUT4 migrates in the cell? Insulin. In a healthy body, when the hormone insulin is present, GLUT4 moves to the cell membrane. When it's not present, GLUT4 retreats to the inner cell. In a nutshell, insulin is the "key" that unlocks the cell doors, allowing glucose to enter. In cases of insulin resistance, GLUT4 transporters don't respond properly to insulin. Even if the hormone is present, they may stay sequestered in the inner part of the cell, leaving the doors locked. As a result, glucose is unable to enter, and blood sugar levels stay elevated. What is unique about banaba leaf is that two of its bioactive compounds - corosolic acid and ellagitannins - "pinch hit" for insulin. Both constituents direct GLUT4 to move to the cell membrane and activate it to incorporate glucose into the cell. Specifically, corosolic acid has been shown to activate GLUT4 in skeletal muscle cells. while ellagitannins have been shown to activate GLUT4 in fat cells. The net effect is that more glucose enters the cells, reducing the level of sugar in the blood. 
- Mulbery leaf Extract: Mulberry (Morus alba) leaf, the food of silkworms, has been used as a healthful food ingredient for humans in India, as well as in Chinese traditional medicine for hundreds of years as a "cooling" herb to remove excessive heats and toxins from the body. However, it was not until recent years that it started gaining attention by doctors and scientists for its promising blood sugar management properties. Studies have shown, among other things, that alkaloids and N-containing sugars isolated from mulberry leaves are potent inhibitors of alpha glucosidase, a key enzyme in carbohydrate and sugar metabolism pathways.Major clinical trials conducted by a team of researchers and doctors at the University of Minnesota (Minneapolis VA Hospital), demonstrate that mulberry leaf, the food source of silkworms, can help markedly stabilize blood sugar levels and inhibitcarbohydrate absorption in Type 2 diabetics by providing additional support which enables them to make better dietary and lifestyle choices. In these studies, a proprietary mulberry leaf extract developed by Lee Zhong, M.D., Ph.D., a graduate and former researcher at University of California, Los Angeles (UCLA), was shown conclusively toReduce body's absorption of sugars and other carbohydrates, Lower post-meal blood sugar spikes and stabilize blood sugar levels: One of these studies published in Diabetes Care, the most widely read diabetes journal, reported an average 44 percent reduction in peak post-meal blood sugar elevations (or spikes).

- 4 Hydroxy isoleucine - (from fenu greek): insulinotropic compound, 4-hydroxyisoleucine. An amino acid has been extracted and purified from fenugreek seeds, which are known in traditional medicine for their antidiabetic properties. 4-Hydroxyisoleucine increases glucose-induced insulin release, in the concentration range of $100 \mathrm{micromol} / \mathrm{l}$ to $1 \mathrm{mmol} / \mathrm{l}$, through a direct effect on isolated islets of Langerhans from both rats and humans. The stimulating effect of 4-hydroxyisoleucine was strictly glucose dependent; indeed, ineffective at low $(3 \mathrm{mmol} / \mathrm{l})$ or basal $(5 \mathrm{mmol} / \mathrm{l})$ glucose concentrations, the amino acid potentiated the insulin secretion induced by supranormal (6.6-16.7 mmol/l) concentrations of glucose. In addition, in the isolated perfused rat pancreas, we could show 1) that the pattern of insulin secretion induced by 4-hydroxyisoleucine was biphasic, 2) that this effect occurred in the absence of any change in pancreatic alpha- and delta-cell activity, and 3) that the more glucose concentration was increased, the more insulin response was amplified. Moreover, 4-hydroxyisoleucine did not interact with other agonists of insulin secretion (leucine, arginine, tolbutamide, glyceraldehyde). This secretagogue thus considered as a novel drug for the treatment of NIDDM.

- EGCG (from green tea): Green tea has been used traditionally to control blood sugar in the body. Animal studies suggest that green tea may help prevent the development of type 1 diabetes, Green tea may help regulate glucose in the body. clinical studies have found that daily supplementation of the diet with green tea extract powder lowered the hemoglobin A1c level in individuals with borderline diabetes. There is also epidemiological evidence that drinking green tea and black tea helps to prevent diabetes .

- Epigallocatechin gallate supplementation alleviates diabetes in rodents. Study shows that EGCG beneficially modifies glucose and lipid metabolism in H4IIE cells and markedly enhances glucose tolerance in diabetic rodents. 
- Bilberry Extract: Bilberry (Vaccinium myrtillus), also known as the Whortleberry and Blaeberry, is a small shrub found on heaths where it grows to about 60 centimeters tall. Bilberry has been used for centuries as a food and a medicinal herb. Bilberry contains substances called anthocyanosides and vitamin $\mathrm{C}$, antioxidants that may help to prevent or reverse injury to cells and manage various illnesses, such as diabetes. Bilberry has been shown to possibly decrease the risk of some diabetic complications, such as diabetic cataracts and retinopathy. One 1 study showed that, bilberry improves signs of retinal damage, such as decreased bleeding in the eye, and strengthened blood vessels.

- Alphalipoic Acid: ALA has been used to improve liver health, treat diabetes-related nerve damage and help regulate blood sugar, prevent diabetic retinopathy (damage to the eyes done by diabetes), and provide protection against oxidative processes involved in thedegenerativediseases.Alpha Lipoic Acid have reported significant reductions in complications associated with diabetes, including neuropathy, and cataracts . ALA has shown benefits in also found reversing diabetic neuropathy and in assisting the body ability to utilize glucose. There is evidence which suggests that ALA my help diabetics reduce their reliance on insulin.Alpha-Lipoic Acid in Diabetic Neuropathy (ALADIN) study evaluated three intravenous doses of alpha-lipoic acid and involved 328 type 2 diabetics with neuropathy. These patients were treated with $1,200 \mathrm{mg}, 600 \mathrm{mg}$, or $100 \mathrm{mg}$ of the antioxidant or a placebo for a period of three weeks. Improvements of $30 \%$ or more in symptom scores were seen in $71 \%$ of the $1,200-\mathrm{mg}$ group, in $82 \%$ of the 600 -mg group, in $65 \%$ of the $100-\mathrm{mg}$ group, and in $58 \%$ of the placebo group.

- $\quad N$-acetyl cystine - An amino acid derivative converted into glutathione (The body's master Antooxidant) in the body. $\mathrm{N}$-acetyl cystine decreases plasma soluble vascular cell adhesion molecule-1 in NIDDM.

- Chromium Picolinate - Chromium Picolinate has been well studied for its beneficial effect on insulin resistance \& diabetes. In addition to improving insulin function it also enhances glucose uptake \& glycogen synthesis.

- Vitamin-B6- has been shown to help protect against diabetic neuropathy\& it also contributes to inhibition of glycation \& those destructive AGE,S.

- Vitamin C- it contributes to inhibition of glycation \& those destructive AGE,S.

- Vitamin $E$ - decreases glucose disposal rate \& no. of insulin receptors on erythrocytes

- Vitamin K- improves insulin sensitivity \& blood sugar regulation.

- Vanadium-improves insulin sensitivity \& blood sugar lowering ability.

- Biotin- helps to lower post prandial glucose level as well as improves insulin sensitivity \& blood sugar regulation. 


\section{INDICATIONS}

A Nutriphenotyphic Approach. Insulike is an Ideal Regimen to fight against the single \& most important metabolic disease which can affect nearly every organ system in the body.

It has the power to protect from metabolic syndrome / type 2 diabetes mellitus. Few ingredients in insulike beneficially influence the expression of your genetic code \& biochemical make up to decrease the disease risk.

Insulike help to support the benefits of controlling Diabetes beyond glucose metabolism., Inhibits glucose absorption, Stimulates insulin Secretion, Improves insulin binding, Improves capillary function, Prevents Pufa peroxidation \& also improves insulin sensitivity.

Contra-indications: Product is contra-indicated in persons with Known hypersensitivity to any component of the product hypersensitivity to any component of the product.

Recommended usage: Adults:1-2 tablet twice a day with water or liquid of choice twice daily

"Do not exceed the recommended daily dose"

Administration: Taken by oral route at any time with food.

Precautions: Do not exceed the recommended daily dose.

Warnings: If you are taking any prescribed medication or has any medical conditions always consults doctor or healthcare practitioner before taking this supplement.

Side Effects: Very Mild side effects like nausea, headache and vomiting in some individuals may be observed.

Storage: Store in a cool, dry and dark place.

\section{SUMMARY \& CONCLUSION}

Insulike as a phenotypic nutrition can help modulate the expression of unique code, thus dramatically reducing your risk of developing disease. Insulike tablet contains all natural nutrients targeted against metabolic syndrome, Type-II Diabetes and Cardiovascular illness, Insulike helps in inhibition of excessive glucose absorption, stimulates insulin secretion, improves insulin binding, capillary function ,Insulin sensitivity, prevents PUFA peroxidation, reduces LDL, VLDL (bad cholesterol) and increase HDL (good cholesterol)

\section{ACKNOWLEDGEMENTS}

Authors are thankful to the Staff \& Management of Lactonova Nutripharm Pvt. Ltd., Hyderabad, Telangana for their cooperation and support .

\section{REFERENCES}

1.PATHOPHYSIOLOGY by Cotran \& Robbins, 2003.

2. J Am Coll Nutr August 2001 vol. 20 no. 4 327-336. 
3. Hayashi T, et al. Ellagitannins from Lagerstroemia speciosa as activators of glucose transport in fat cells. Planta Medica. 2002 Feb;68(2):173-5.

4. Diabetes February 1998vol. 47 no. 2 206-210.

5. Life sci kgg; 42;1323-1330

6. Chem. Res 2,$4 ; 237-48,2002$

7. Diabetes Res. Clin pact 1994; 44:27-33.

8. Juturu V, chromium \&insulin resistance, Experimental biology2004, 351-7.

9. Diabetolgiab1998 Nov.41 (11):1392-136

10. Van Dam, P.S. "Oxidative stress and diabetic neuropathy: pathophysiological mechanisms and treatment perspectives." Diabetes Metabolic Research Review. 2002 May-Jun;18(3):176-84.

11. Diana Kaniecki University of Maryland Medical Center: Bilberry.

12. J Nutr. 2006.

13. Iso $\mathrm{H}$ et al. (2006). The Relationship between Green Tea and Total Caffeine Intake and Risk for Self-Reported Type 2 Diabetes among Japanese Adults. 144. pp. 554-62.

14. Diabetes Res Clin Pract. 2006;71(3):356-8.

15. Ryu OH, Lee J, Lee KW, et al. Effects of green tea consumption on inflammation, insulin resistance and pulse wave velocity in type 2 diabetes patients. Diabetes Res Clin Pract. 2006;71(3):356-8.

16. Diabetes February 1998vol. 47 no. 2 206-210.

17. Planta Medica. 2002 Feb; 68(2):173-5. 